\title{
Resource Prospector (RP) - Early Prototyping and Development
}

\author{
D. Andrews ${ }^{1}$ \\ NASA-Ames Research Center, Moffett Field, CA, 94035, USA \\ A. Colaprete ${ }^{2}$ \\ NASA-Ames Research Center, Moffett Field, CA, 94035, USA \\ J. Quinn ${ }^{3}$ \\ NASA-Kennedy Space Center, Kennedy Space Center, FL, 32899, USA \\ B. Bluethmann ${ }^{4}$ \\ NASA-Johnson Space Center, Houston, TX, 77058, USA \\ and \\ J. Trimble ${ }^{5}$ \\ NASA-Ames Research Center, Moffett Field, CA, 94035, USA
}

\section{Abstract}

$\mathrm{T}$ he Resource Prospector (RP) ${ }^{[1]}$ is an In-Situ Resource Utilization (ISRU) technology demonstration mission under study by the NASA Human Exploration and Operations Mission Directorate's (HEOMD) Advanced Exploration Systems (AES) Division. The mission, currently planned to launch in 2020, will demonstrate extraction of oxygen from lunar regolith to validate ISRU capability. The mission will address key Strategic Knowledge Gaps (SKGs) for robotic and human exploration to the Moon, Near Earth Asteroids (NEAs), and ultimately Mars, as well as meet the strategic goals of the Global Exploration Roadmap (GER), offered by the International Space Exploration Coordination Group (ISECG). In this roadmap, the use of local resources is specifically addressed relating to human exploration ${ }^{[2]}$

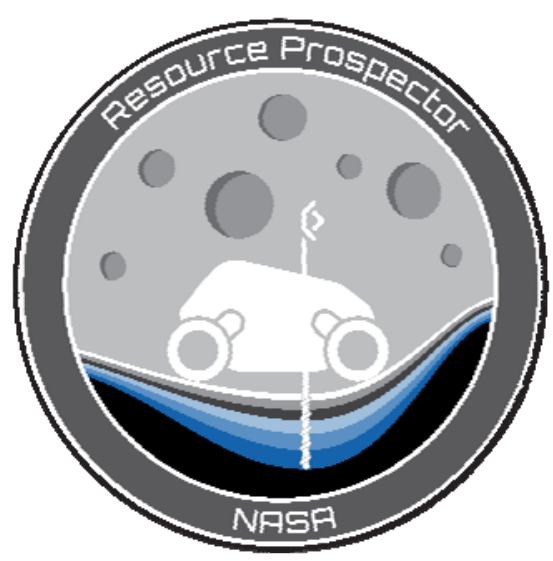

Figure 1. Resource Prospector team

RP will provide knowledge to inform the selection of future mission destinations, support the development of exploration systems, and reduce the risk associated with human exploration. Expanding human presence beyond low-Earth orbit to asteroids and Mars will require the maximum possible use of local materials, so-called in-situ resources. The moon presents a unique destination to conduct robotic investigations that advance ISRU capabilities, as well as providing significant exploration and science value. Lunar regolith contains useful resources such as oxygen, water, silicon, and light metals, like aluminum and titanium. Oxygen can be separated from the regolith for life support (breathable air), or used to create rocket propellant (oxidizer). Regolith can be used to protect against radiation exposure, be processed into solar cells, or used to manufacture construction materials such as bricks and glass. RP will characterize the constituents and distribution of water and other volatiles at the poles of the Moon, enabling innovative uses of

\footnotetext{
${ }^{1}$ RP Project Manager, Programs \& Projects Directorate, Moffett Field, CA 94035

${ }^{2}$ RP Project Scientist, Planetary Systems Branch, Moffett Field, CA 94035

${ }^{3}$ RP Payload Project Manager, Surface Systems Office, KSC, FL 32899

${ }^{4}$ RP Rover Project Manager, Software, Robotics and Simulation Division Office, Houston, TX 77058

${ }^{5}$ RP Mission Operations Manager, Exploration Technology Directorate, Moffett Field, CA 94035
} 
local resources, in addition to validating ISRU capabilities. This capability, as well as a deeper understanding of regolith, will be valuable in the exploration of near-Earth asteroids (NEAs) and Mars.

In order to reduce risk and explore system designs, the RP project is attempting two-fold approaches to development as it looks towards flight. We continue to explore flight planning, requirements, and interfaces definition by using Engineering Test Units (ETUs), looking towards lunar deployment, while also using fiscal year 2015 to develop, build and test an earth-terrestrial prototype rover and payload system. This terrestrial prototype, called "RP15", is built to both inform the system design, and to be a partnership advocacy tool for this unique mission.

RP15 must be affordable within the resource and time constraints of fiscal year 2015, while working to the following Needs, Goals, and Objectives provided by HEOMD/AES:

1. Demonstrate rover mobility in a $1 \mathrm{~g}$ environment

2. The Surface Segment (prototype rover + payload system) shall represent the flight system concept with as much fidelity as affordable (limited by cost and schedule)

- Surface Segment shall be the approximate size/dimension/footprint

- Surface Segment shall package all the expected devices (instruments, systems, etc.), even if some facets are mocked-up due to time/cost constraints

- Overall Surface Segment fidelity negotiable to make achievable

3. Priority should be given to illustrating mission functionality over support functionality, which exists solely to support mission functionality

This paper will provide an overview of RP project developments, including the design and build, capturing the development and initial integrated testing of RP15 in relevant environments.

\section{The Two-Fold Nature of Resource Prospector Development}

Resource Prospector is a Phase A project, managed within the Advanced Exploration Systems (AES) Division of NASA's Human Exploration and Operations Mission Directorate (HEOMD), and planning for launch in 2020. The activities which apply to the RP flight plans and hardware are collectively referred-to as "RP" - the mission which will fly. Additionally in the 12-month span of fiscal year 2015, the RP team has stepped-up to the challenge of building a terrestrial Engineering Test Unit (ETU) surface segment to be used to mature technologies, perform risk reduction, and practice how we would operate the actual mission. This ETU rover/payload surface segment will be taken from conceptual Needs, Goals, and Objectives (NGOs) to a working rover/payload ETU in a single year. This great challenge of building this ETU will promote learning and enable risk reduction activities to take place, while still in Phase A, and is referred-to as "RP15".

Both RP15 and RP seek the same functionality in general; however, RP15 is limited by both programmatic and terrestrial constraints. In general, RP is specifically designed to prospect the lunar surface, create a map revealing the nature and distribution of the volatiles, and perform an early demonstration of processing while on the lunar surface. RP15 enables early testing of some of the most important capabilities required to execute the flight plan of RP. More specifically, RP15 will:

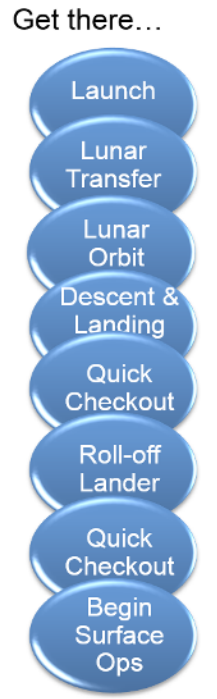

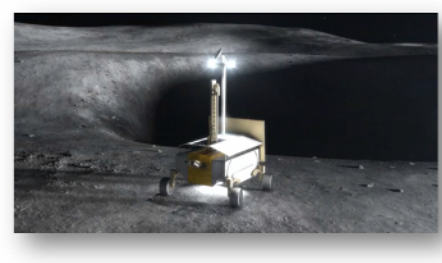

Find \& Excavate Volatiles...

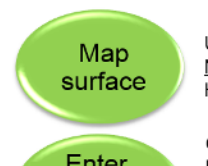

Enter permanent vighest concentrations of shadows Shadowed Regions shadows (PSRs)

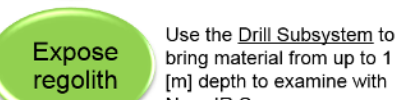
regolith $[\mathrm{m}]$ depth to examine with Near-IR Spec
Collect and Process the volatiles...

\section{Capture Use the Drill Subsystem to} regolith capture samples from up to $1[\mathrm{~m}]$ depth

regolith
Heat regolith

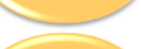

Identify Volatiles LAVA Subsystem, $(\mathrm{H} 2, \mathrm{He}$ $\mathrm{CO}, \mathrm{CO} 2, \mathrm{CH} 4, \mathrm{H} 2 \mathrm{O}, \mathrm{N} 2$, $\mathrm{NH} 3, \mathrm{H} 2 \mathrm{~S}, \mathrm{SO} 2$ )

Show Image and quantify the water! LAVA Subsystem

Figure 2. RP Simplified overview 
1. Demonstrate rover mobility in a $1 \mathrm{~g}$ environment

2. Represent the flight system surface segment with as much fidelity as affordable (limited by cost and schedule)

3. Give priority to illustrating mission functionality over support functionality, which exists solely to support mission functionality

RP functionality is illustrated in Figure 2, and some of the functionalities are areas in which RP15 will reduce risk by doing early Phase-A testing.

1. Mapping the Surface. The RP15 terrestrial rover will provide mobility enabling roving over surfaces and slopes analogous to what will be expected on the polar regions of the moon. The force of gravity is different for our RP15 terrestrial ETU, but is still relatable to what will be experienced on the moon. Further, the rover system is being designed to enable testing in gravity off-load facilities to see how it performs in a true $1 / 6 \mathrm{~g}$ environment. This roving platform will carry both the Neutron Spectrometer System (NSS) and Near Infrared Volatiles Spectrometer System (NIRVSS). During RP15 field testing the NSS is included to replicate

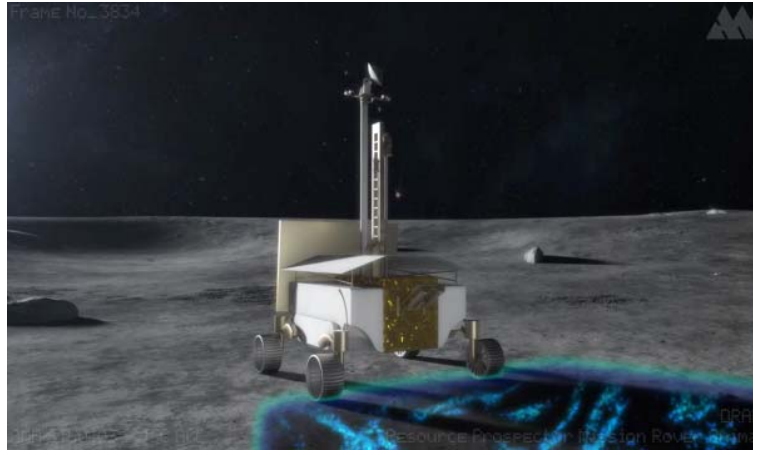

Figure 3. RP scanning for subsurface volatiles packaging constraints, but will not be functional; however, the NIRVSS instrument will be functional, enabling sensing/measuring the indigenous soil and practicing the prospecting part of the mission. Figure 3 illustrates how this scanning would work during actual lunar roving.

2. Entering Permanent Shadows: We envision RP15 will navigate terrestrial "Permanently-Shadowed Regions" (PSR) by either testing at night in the rock yard, or by testing in a high-bay with darkened conditions. The degree of fidelity (regolith simulant, volatile doping, etc.) is negotiable based on resources available, but this testing could aid in understanding navigation, positioning, and measurement difficulties in roveronly lighted conditions. Figure 4 illustrates the RP rover charging in the Sun prior to entering a PSR on the moon.

3. Exposing Regolith: The Drill system is also included

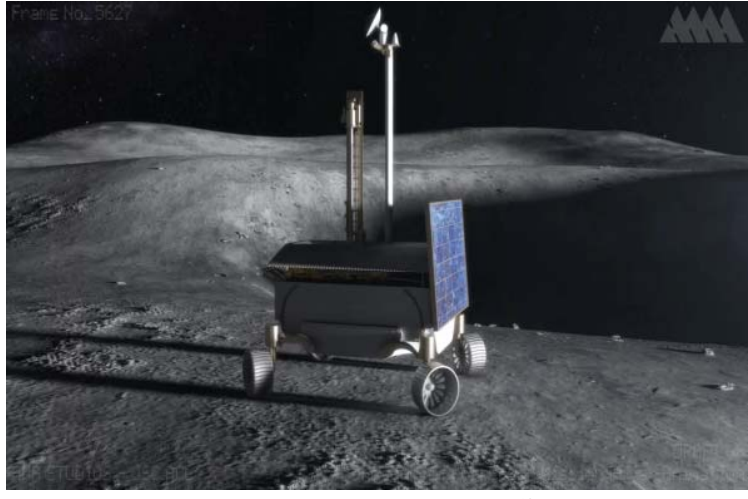

Figure 4. RP prior to entering a PSR

on the RP15 ETU, enabling actual drilling operations from the rover. This will enable testing procedures and methods for drilling and capturing tailings from the drill bit, even while acknowledging this testing will be in a $1 \mathrm{~g}$ environment. We expect to learn about constraints on the rover system while drilling on slopes, which side-loads the drill bit, potentially causing binding. The rover suspension system will be required to compensate for loads which move during these drilling conditions, enabling extrapolation of what might be found in the $1 / 6 \mathrm{~g}$ environment of the moon. Further, the NIRVSS instrument will measure the volatiles excavated from the soil to the surface. We envision having specifically-prepared sample tubes, perhaps buried in the lunar rock yard, wherein the rover can drive over the sample tube, drill/excavate and measure.

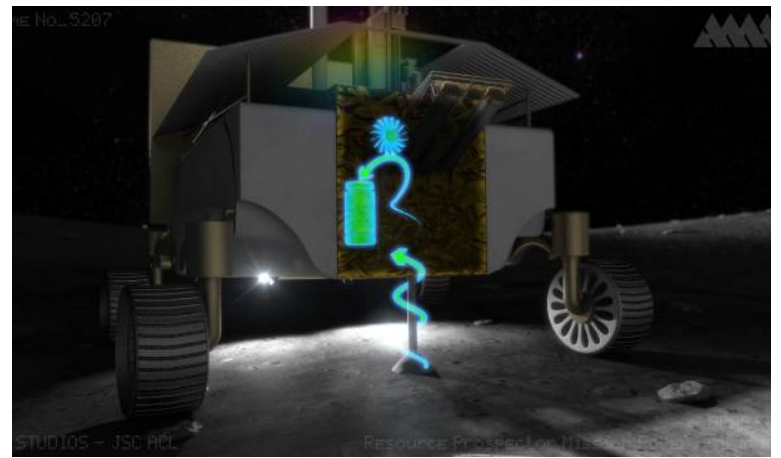

Figure 5. RP drilling for subsurface volatiles 
4. Capturing and Heating Regolith: The same drill which provides the excavation capability illustrated above also has the capability to acquire samples from as deep as $1 \mathrm{~m}$, into a sample handling system. The samples are then deposited into the Oxygen and Volatile Extraction Node (OVEN) Subsystem, evolving the volatiles by heating the regolith in a sealed chamber and extracting oxygen and hydrogen from the regolith sample. RP15 will be carrying an ETU version of OVEN, capable of performing similar functions to the flight version of the same subsystem. This includes the ability to heat the regolith samples to 150-450 degC to liberate the volatiles for measurement. Figure 5 illustrates the RP rover performing a drill operation and bringing material from $1 \mathrm{~m}$ below the surface up into the OVEN system.

5. Identifying the Volatiles: The RP Lunar Advanced Volatile Analysis (LAVA) Subsystem is also included in on RP15. This system will analyze the effluent gas/vapor from the OVEN Subsystem using gas chromatograph and/or mass spectrometer sensor technologies. Volatiles evolution from material in the doped sample tubes will enable verification of the design approaches planned for RP.

\section{RP15 Planning}

RP15 planning was complex, given the extraordinarily short amount of time to turn-around a functioning ETU in a single year. The RP Project Manager (PM) decided that this RP15 opportunity could carry benefits even beyond the build and test of an ETU system. It could also be an opportunity to train in flight-like behavior, given there are many on the team who have not worked on a spaceflight project before.

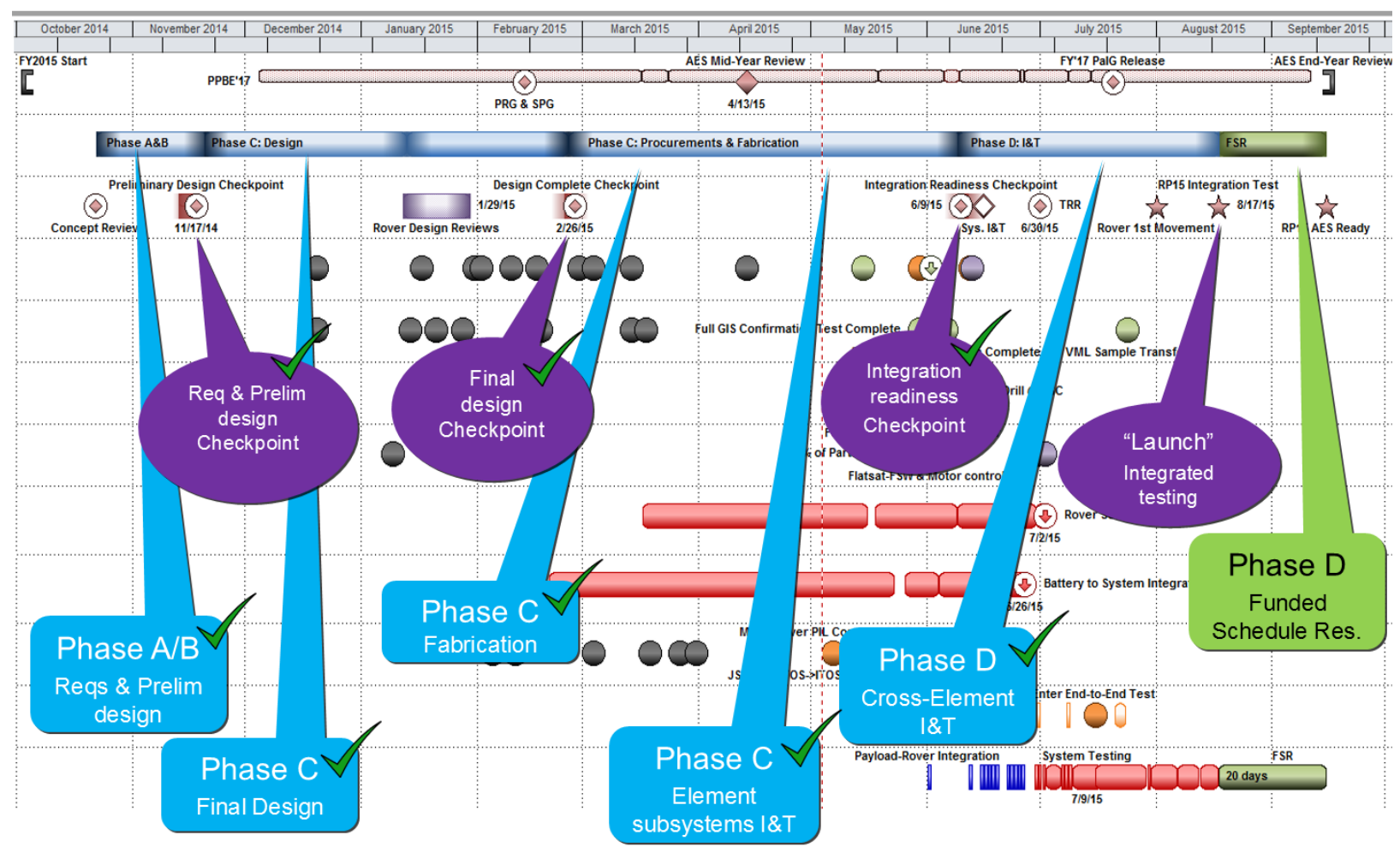

Figure 6. RP15 “mission-in-a-year" schedule covering the entire NASA Project lifecycle

Some examples of RP15 training for the future flight mission:

- Integrated Master Schedule (IMS) creation and tracking

- Reserves Management and tracking (Schedule and Cost)

- Risk/Threats management (streamlined)

- Requirements management (specifications and ICDs)

- Analogous Gate reviews required for flight (tailored)

4

American Institute of Aeronautics and Astronautics 
- Procurement planning

- Scope management and contingency planning (fitting in the bag or multiple, smaller bags)

- Assembly, Integration \& Test practice (planning and execution) on highly relevant hardware

The RP15 team started by creating a "mission-in-a-year" schedule, walking through the entire project life cycle! Figure 6 reveals the RP15 plan with all the normal project phases shown and equivalent gates as would be required for flight. Looking carefully at the schedule shows how quickly some of the phases occurred: Preliminary design was completed in 1.5 months, with final design complete 3.5 months later. RP15 then completed its procurements and fabrications in a couple months and then started building sub-systems, which a month later got assembled. The whole ETU system-level assembly completed in about two months enabling integrated testing to commence, or "Launch".

As noted above, part of the benefit of RP15 is the designing, building and testing against a tailored flight-like construct, but this activity also includes behavioral training. For example, the RP15 Project carried Funded Schedule Reserve (FSR) commensurate with the levels you'd find on a flight project, computed based on durations in each of the project lifecycle phases. The same is true with the Project Unallocated Future Expenses (UFE - or reserves). The PM held both FSR and UFE in a war chest, not to be consumed until the design was finalized. This was done to assure that the scope of the project matched the available resources; there would be no pre-liens against the reserves in the design. The reserves existed to address unknowns and generally-accepted risks in the design and build of the hardware and were formally tracked by project management. A simplified process was created to identify, monitor, and document risks, the potential threat to FSR and UFE, and make requests for FSR and UFE. The project captured its risk in a time-efficient manner using a Threats/Lines list, which captured the nature of the risk; whether the risk was a threat to FSR, UFE, or both; and when in the 12-month horizon the project might realize the threat. The PM adjudicated based on input from the team, cost-to-go, and schedule-to-go information to retire, lien or continue monitoring the risks. This approach proved to be a very lightweight process in which the team leads would engage, with the PM making the final decision on expenditures.

RP15 technical performance requirements originated from the RP flight requirements. To minimize confusion between the two sets of requirements (since the project team is working on both RP15 and RP activities in 2015), the RP15 requirements were referred-to as "Specifications". The RP requirements are captured in DOORS, while the RP15 specifications are kept in a configuration-controlled spreadsheet on a team server. The Project took the RP flight requirements and tailored, simplified, and in some cases deleted requirements when creating the RP15 specifications. The beauty of this approach is that there is documented trace to which flight requirements are getting "touched" by the RP15 activities. In a few cases the touch was non-existent, while in most cases, loosened-versions of the requirement were levied on RP15 as a specification.

\section{RP15 Payload Development}

The RP Payload is based on RESOLVE (Regolith and Environment Science and Oxygen and Lunar Volatile Extraction), developed in previous years as a candidate ISRU instrument suite. The RP team identified several key driving requirements and risks it wished to explore or retire during RP15 activities. These requirements were contingent upon operating on a mobility platform (rover), and involved the coordination between two or more of its onboard instruments. Specifically, the team sought to focus on three key areas for requirements development and risk reduction: Near Surface Assay coordination with Drill, Drill sample delivery to OVEN, and drilling on slopes and under reduced gravity conditions. 
Near Surface Assay activities were chosen to improve efficiencies and retire the loss of volatiles risk, while minimizing mission time impacts. Onboard software was employed to operate the drill and NIRVSS systems in a rapid assessment of short $(10 \mathrm{~cm})$ "pecks" or "bites" of regolith that are progressively brought to the surface from the same hole ${ }^{[3]}$. The drill augers down $10 \mathrm{~cm}$, captures material on its flutes before rising out of the hole, and the NIRVSS system rapidly acquires a visual spectrum estimating water abundance real-time. The drill then augers further down the same hole, picking up regolith on its flutes from the $10-20 \mathrm{~cm}$ below grade depth before returning again to the surface for NIRVSS spectrum acquisition and potential water identification/quantification. This process is repeated until the maximum drill depth of $100 \mathrm{~cm}$.

Further risk retirement is possible by testing the delivery of regolith from the drill flutes into this OVEN system; drill and crucible testing can be seen in Figure 7. Within OVEN, the granular regolith substrate is heated to drive off the loosely bound and surficially bound water for analysis by the LAVA system. If the Near Surface Assay indicates a reasonable water signature, a real-time operations call would initiate a volatiles analysis sequence, requiring a drill sample delivery to the OVEN and subsequent qualification/quantification of water by LAVA's Gas Chromatograph/Mass Spectrometer instrumentation. This coordination, or "ballet" of relational activities will be tested in RP15 for both technical efficiencies as well as mission

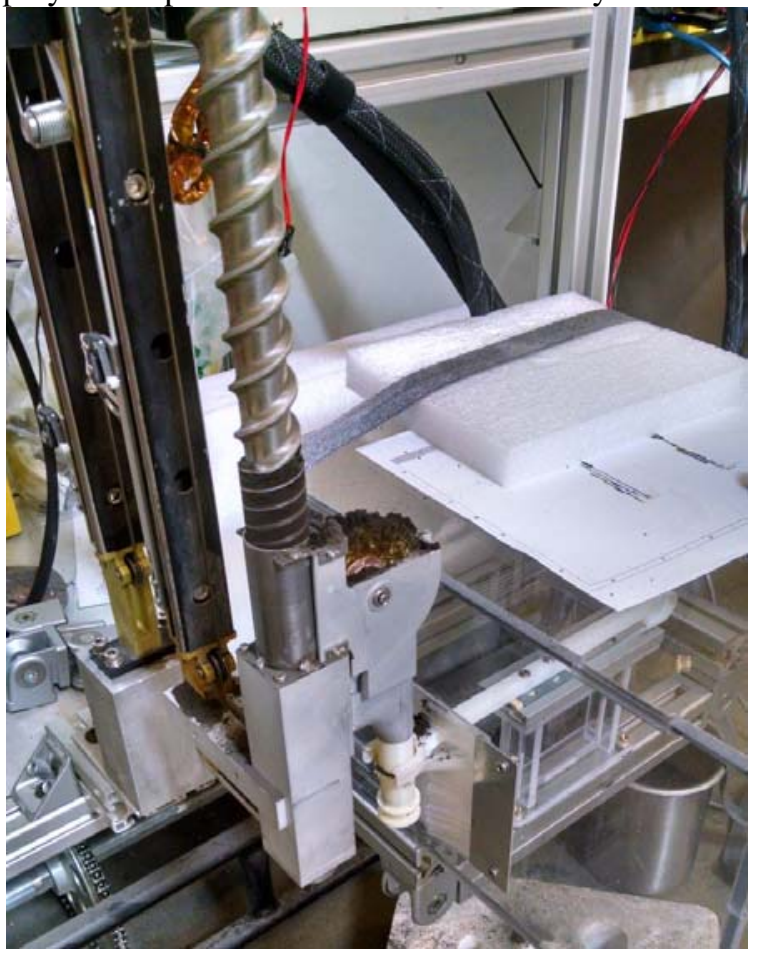

Figure 7. RP15 Drill/Crucible testing timeline implications.

The payload team also sought to understand the limitations imposed on drilling and sample acquisition while operating on slopes. Further, the impact to the rover structural stability as well as the drill's stability and ability to maintain alignment with the OVEN system will be tested both in earth's gravity and in lunar gravity.

The development process for designing and building the RP15 payload system was both schedule and budget driven. Since some payload instruments existed from previous work, use of current hardware was determined to be the lowest risk. In some instances, hardware was taken out of thermal vacuum chambers and reconfigured to run in a terrestrial environment. The payload team used flight-like processes during the Assembly, Integration and Testing phase of RP15, utilizing procedures such as Work Order Authorizations and Anomaly Reports, thereby increasing the development team's flight readiness. Within the RP15 year of execution, the RP team began working to develop flight-like procedures for the payload's execution. This included flight-forward ground data systems components, such as displays, CCSDS (Consultative Committee for Space Data Systems) gateways and voice over IP commanding from a three NASA Centers, distributed operations platform.

To minimize risk of late installation and to allow for the needed rover development/build cycle, a Ground Interface Structure (GIS) was built to simulate the rover payload bay, as shown in Figure 8. A three dimensional CAD model was developed to ensure that the GIS was a 3-D replica of the physical interface of the payload components to the rover. Much, but not all of the payload instrument suite was mounted to the GIS at the Kennedy Space Center and underwent functional testing prior to shipping on the GIS to the Johnson Space Center for later installation onto the Rover platform.

RP15 provided a unique opportunity to mature the software solution that would eventually evolve into the payload flight software deliverable, in Figure 9. In contrast to previous software solutions, which were delivered as a monolithic executable product, RP pursued a federated approach that included individual executable products, each product focused on an individual payload task. The amalgam of these individual products behaved as a cooperating ecosystem, using the Data Distribution Service (DDS) as the main method of communication. Some of the 
executable products acted as software agents, representing individual instruments in the ecosystem. Other tasks

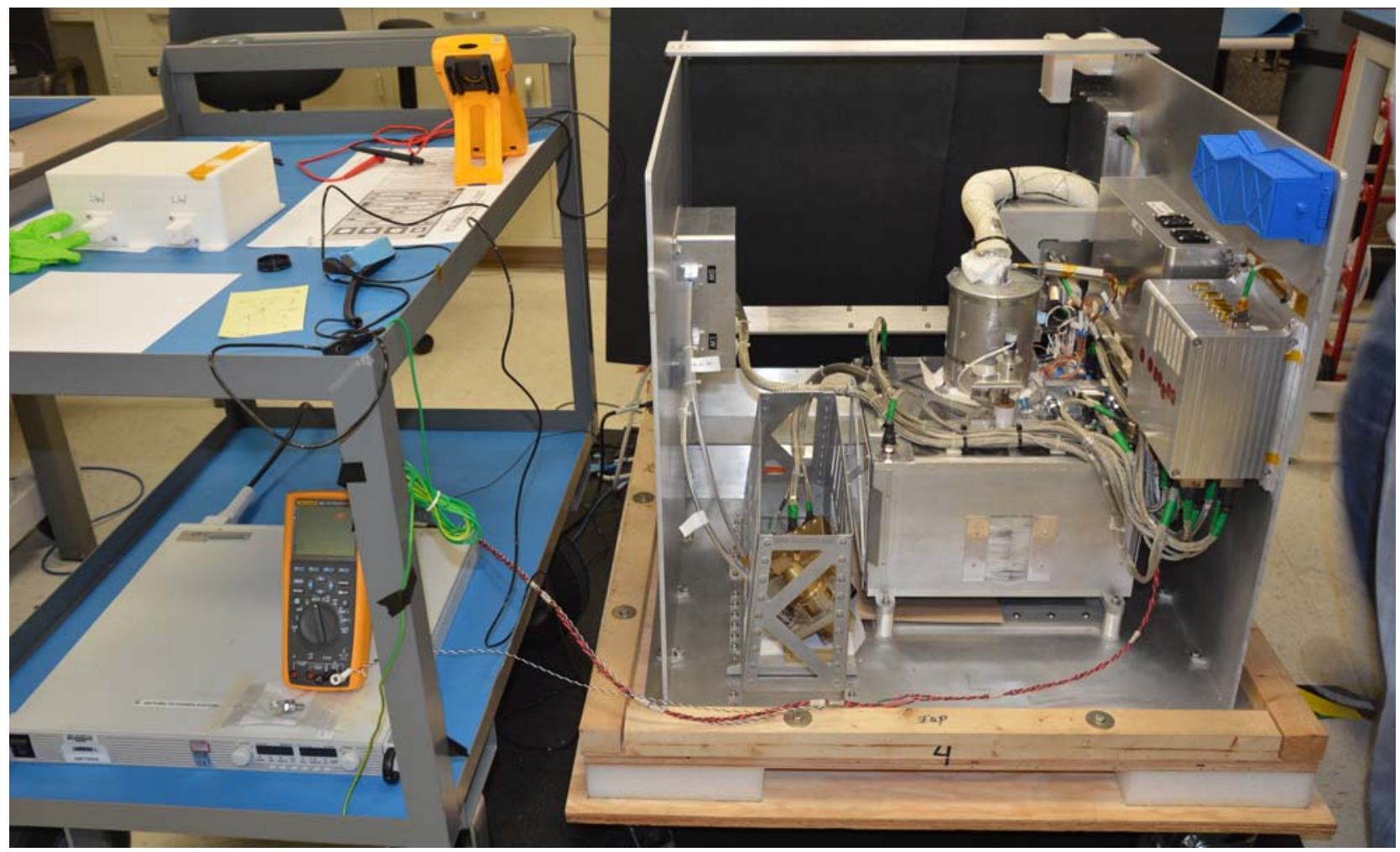

Figure 8. RP15 Payload built and installed in the GIS, ready to ship for Rover integration

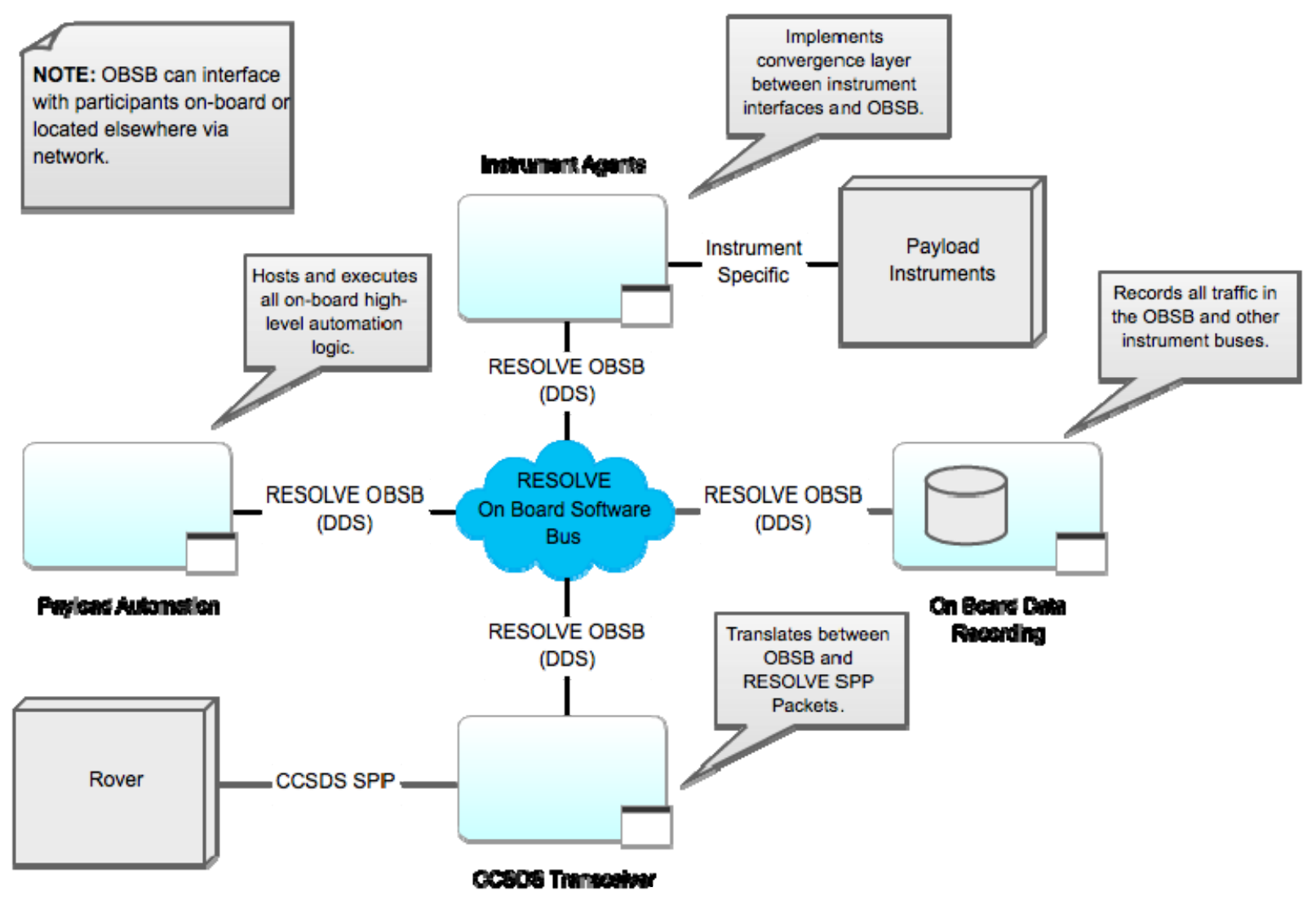

Figure 9. RP15 Payload Software Architecture

American Institute of Aeronautics and Astronautics 
accomplished by the executable products included internal data recording, ground communications, and instrument automation. Automation was implemented using the widely successful Virtual Machine Language (VML); a product originally developed for the NASA Jet Propulsion Laboratory, and now brought to the commercial realm by Blue Sun Enterprises. VML has been instrumental in ensuring success in over a dozen previous NASA missions. DDS has proven to be a popular communications platform, widely used by the United States Armed Forces, among other organizations delivering mission-critical products.

RP15 has enabled behavior in a more flight-relevant framework, as opposed to research-relevant processes, ensuring a smoother entry into Phase B of the flight development cycle. During RP15, older avionics equipment were used for schedule convenience, but continued to develop the software platform. Given that software development is the greatest schedule risk for flight projects, this early investment certainly has significant risk reduction implications for RP.

\section{RP15 Rover Development}

The NASA developed rover for the RP mission began in October 2014; this late start was due to changes in partnering on RP, leading to the rover being developed in-house within NASA. This late start became a notable challenge having to cover much more road in the development and build of the RP15 ETU. Technology development work for the rover is funded through the Game Changing Development (GCD) Program within NASA's Space Technology Mission Directorate (STMD). The work on the rover will leverage prior investments from the agency through the GCD Human Robotic Systems project in areas including, mobility systems, active suspension, motor control, infrastructure, free localization and navigation, and battery systems. Work on the RP rover transitions and matures previous work performed on the Chariot, Centaur, K-10 and KRex research and development rovers towards a flight system ${ }^{[4][5]}$.

For the RP15 rover design, the specifications derived from the RP system requirements were levied onto the rover, while maintaining their linkage to the flight requirements. From these specifications, the RP15 rover derived a few internal driving functional specifications, providing an opportunity to explore risks and technical questions, as illustrated below:

1. The rover will operate outdoors on earth. This is a practical reality of the test environment for terrestrial testing. This encompasses having structures that support $1 \mathrm{~g}$ loads while roving, and scaling actuators to be capable supporting the difference in rover weight. The thermal system utilizes heat spreaders and mockups of a radiator and heat pipes to look like flight. The rover will use fans to cool components convectively for this earth testing.

2. The rover will operate untethered. The rover will have a $5.5 \mathrm{kWh}$ battery to support science operations and roving. The battery architecture is based on the Robonaut 2 battery designed for use on the International Space Station ${ }^{[6]}$. Development of a battery with a path towards flight reduces technical risk on battery management and helps derive requirements for the level of safety required for a robotic mission versus the Robonaut battery in which the design was based. Additionally, the battery will allow the RP team to begin correlating models for energy usage and then extrapolate the results to the lunar environment.

3. The rover will be capable of being remotely driven. RP15 has two options for remote driving. The primary mode is waypoint driving where the operators are in a remote control center. This allows the RP team to explore the surface concepts of operations for the mission. Additionally, the rover can support joystick-controlled local operation wherein an operator, with eyes on the rover, issues body velocity commands. This operation mode allows for the RP rover team to explore core rover functionality independent of the remote waypoint driving.

4. The rover will be capable of carrying a science payload of up to $90 \mathrm{~kg}$. This will enable prospecting, drilling and material processing in the RP15 payload subsystem. In prior versions of the RP roving surface segment, an international partner was building the rover, and the science payload was integrated as a complete unit into the rover. This modular design kept interfaces clean across agencies; however, the price 
of having a clean interface was increased system mass. With the science payload and rover both being developed within NASA, a more integrated, mass-efficient rover/payload design was enabled.

5. The rover will be capable of operating in an exterior "Rock yard". The rock yard at NASA-JSC is a small $\left(\sim 5500 \mathrm{~m}^{2}\right)$ analog test site. Within this rock yard is a lunar area with three craters, a mars area with a variety of rocks ranging in size from $0.05 \mathrm{~m}$, to $1.0 \mathrm{~m}$ in height, a $5 \mathrm{~m}$ tall hill, and an open area capable of terraforming for test specific needs. The slopes in the rock yard vary from flat to up to 20 degrees. In the permanent areas of the rock yard, the surface composition consists of stabilized limestone covered with gravel and rocks of varying size. The RP15 specifications are more ambitious than the flight requirements for slope characteristics, primarily to be able to perform well in the rock yard. The rover is capable of operating in the rock yard during both daytime and nighttime conditions.

6. The rover will have the look and feel of the flight rover. In the spirit of looking toward flight, efforts were made to minimize mass, power and volume (within reason). This led to building non-functional stand-ins for multiple systems, including radiator, heat pipes, and solar array. In keeping with this requirement, the wheels of the rover are sized for a lunar gravity (approx. 1/6 earth's gravity) with 0.8 psi ground pressure. During testing, to achieve similar performance as anticipated on the lunar surface, materials will need to be compacted to support traction studied.

RP15 is designed to be gravity-offload compatible; that is, able to be supported within a gravity equilibration system to better simulate partial gravity environments, like on the moon. This type of work is out of scope for 2015, but is anticipated to be a candidate for a 2016 testing campaign.

To meet the challenges illustrated above, the rover is a four-wheeled vehicle, with the evolution of the design shown in Figure 10. The mobility of the vehicle is composed of hub motor drive, offset four wheel steering, and active suspension. The active suspension provides considerable capability and flexibility to the mission, including the following:

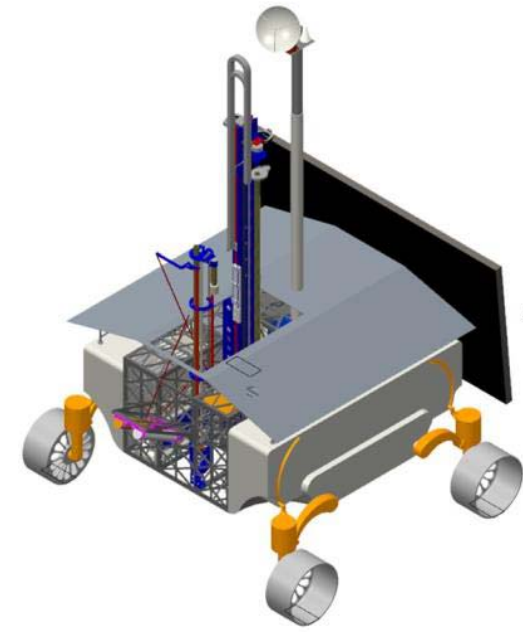

Early Concept: $12 / 2013$

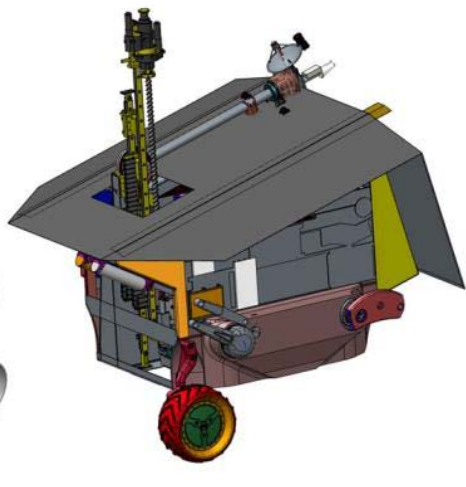

Peer Review: 01/2015

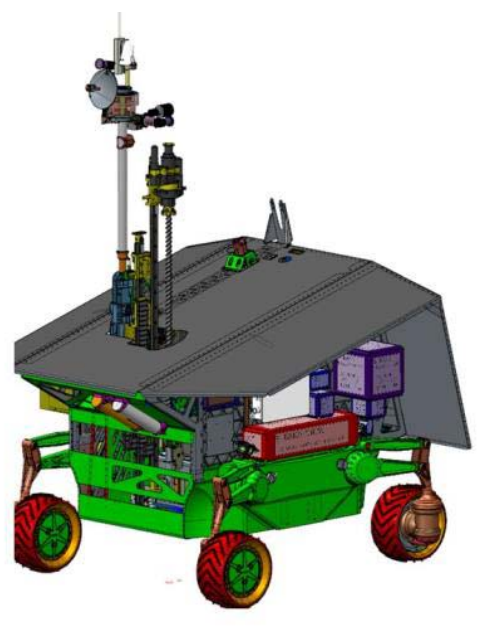

Final Design: 04/2015

\section{Figure 10. Evolution of the RP Rover Design}

- Providing the ability to stow during transit to the moon

- Changing vehicle ride height to avoid obstacles

- Control the center of gravity while operating on slope

- Adjust vehicle angle for drill placement on sloped surfaces,

- Aligning the solar panel to maximize solar flux

- Enabling the ability to traverse more complex through managing normal force on the ground individually 
All-wheel steering provides the ability of the vehicle to point arbitrarily while roving. This enables functionality such as roving while keeping the solar array pointed at the sun and positioning science instruments optimally while in motion. The offset steering when combined with the active suspension provides the ability of the rover to change individual wheel track when stuck in soft soil.

RP15/RP flight software utilizes NASA's Core Flight Software (CFS), a platform-independent, missionindependent, reusable Flight Software environment consisting of the core Flight Executive (cFE), Operating System Abstraction Layer (OSAL), and cFE compliant CFS Applications, illustrated in Figure 11. CFS allows re-use and leverages existing core spacecraft functions while enabling the development of custom applications specific to the application ${ }^{[7]}$. During 2015, FSW build 1 was released, which provides hardware interfaces, basic mobility, waypoint driving, odometry and inertial measurement unit (IMU) based localization, basic error checking, and camera services.

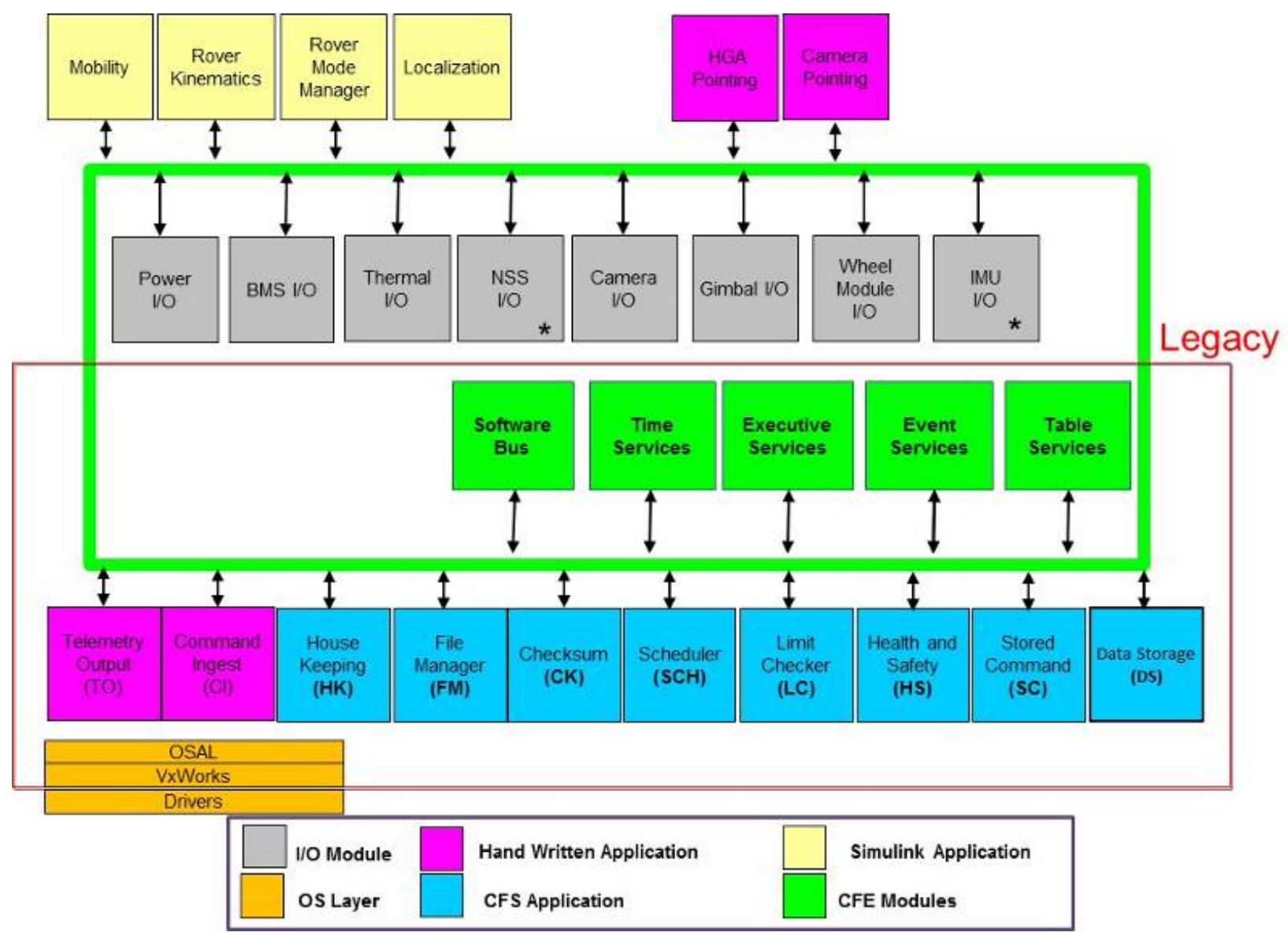

Figure 11. CFS/cFE Architecture for RP15 Rover 


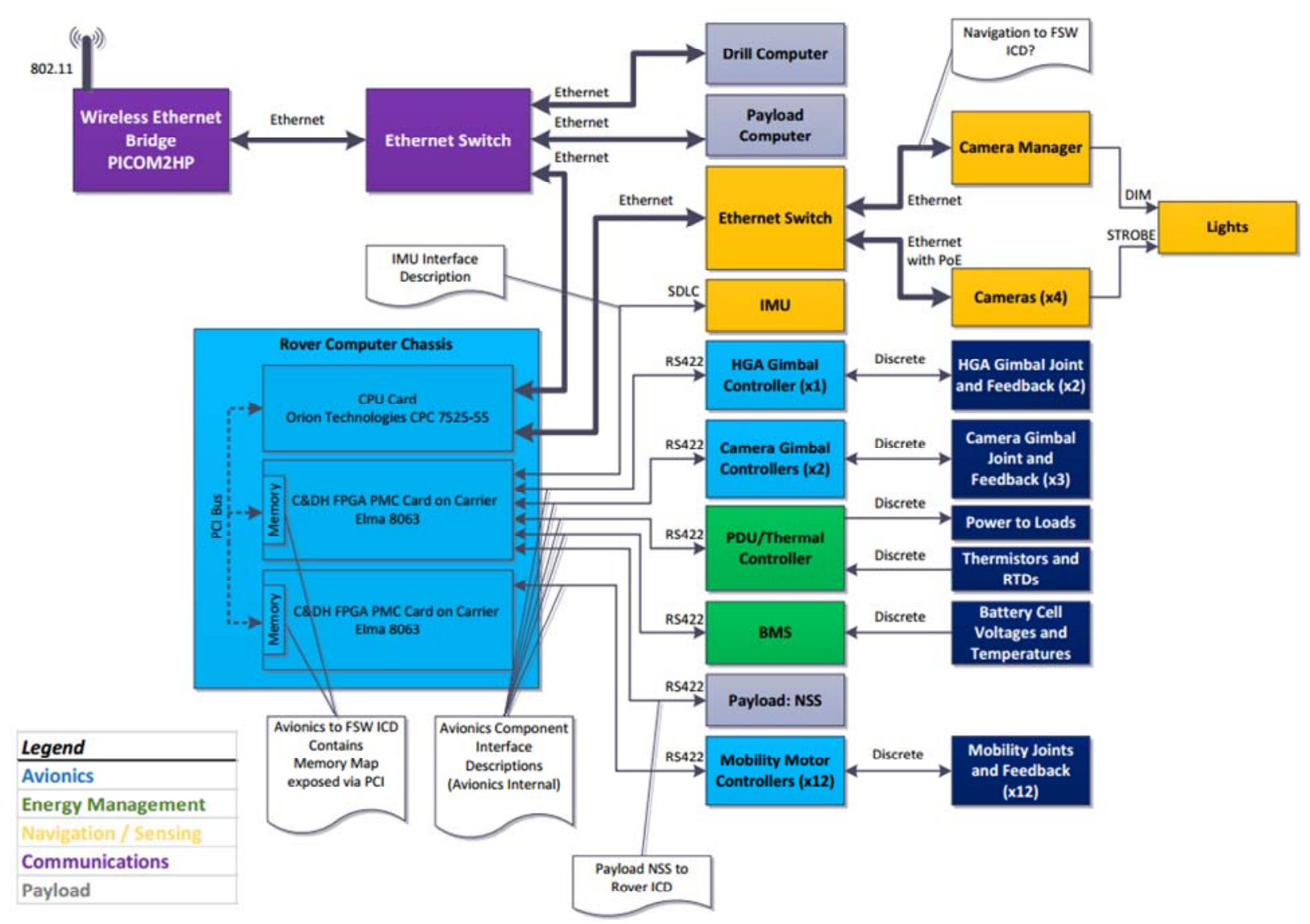

Figure 12. RP15 Rover Avionics Architecture

Avionics on the RP15 rover employ existing designs for the mobility motor control, new designs for power distribution and pointing cameras, and COTS convectioncooled Power PC computer running vxWorks, as illustrated in Figure 12. The mobility motor controllers are the same controllers used by the Robonaut system on the International Space Station and have been through both proton testing at the Indiana University Cyclotron Facility and heavy ion testing at the Brookhaven National Laboratory. The new designs use circuits and approaches that are applicable towards flight, but were forced to add accommodations for RP15 specific functions such as powering fans and Ethernet switches and COTS cameras.

Hardware development work has been fast-paced with

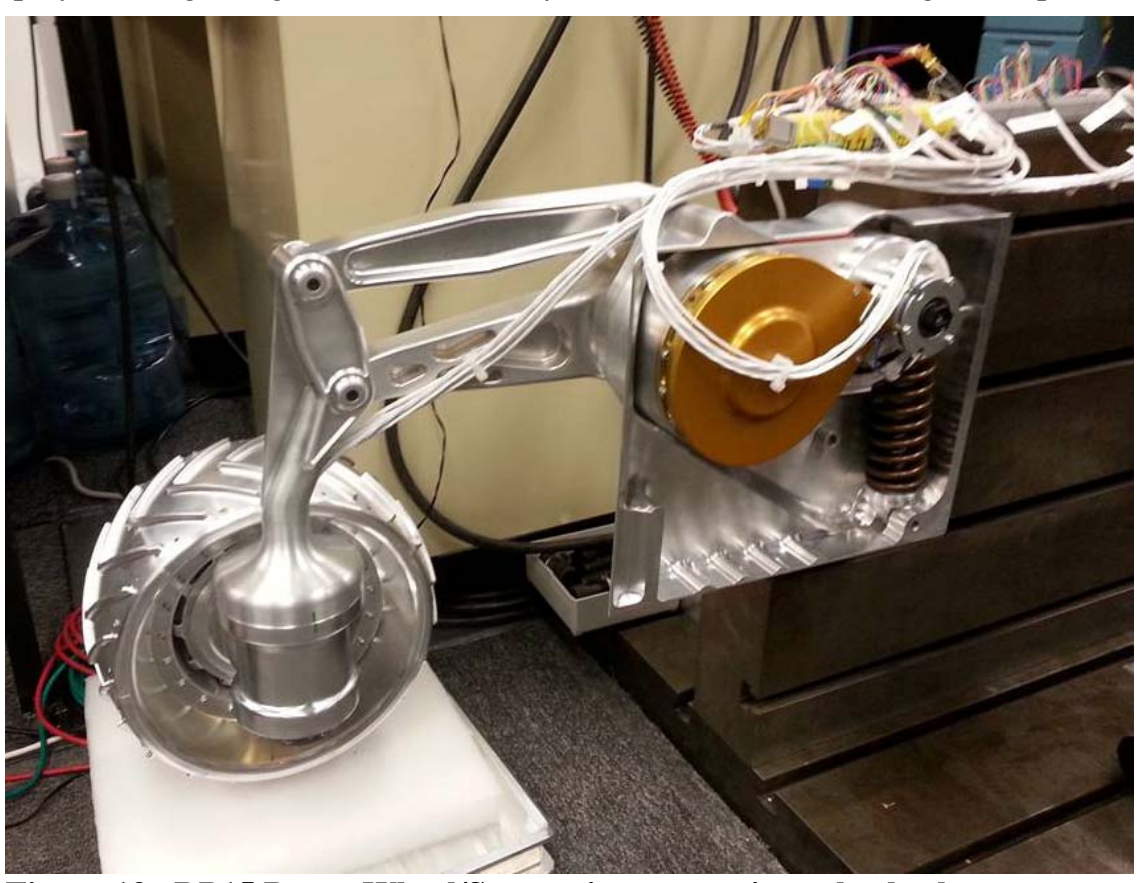

Figure 13. RP15 Rover Wheel/Suspension system in early checkout

American Institute of Aeronautics and Astronautics 
incredible progress made in a very short amount of time. Figures 13 \& 14 illustrate subsystems manufacturing and development leading to the rover build

The RP15 approach matured many of the subsystem designs, has produced the first Flight Software build, and has answered about the approach of a four wheeled rover with active suspension and offset steering. The integrated RP15 ETU also provide the RP project an asset that allows testing of relatively high fidelity concepts of operations going into phase $\mathrm{B}$ of the mission. Lastly, the 2015 development sets the stage for more detailed testing during 2016. This environment testing will include radiation testing on new avionics designs, thermal and thermal/vacuum tests on mechanisms, gravity offload testing to test mobility, and sensing/navigation risk reduction.

\section{RP15 Operations Overview}

RP15 provides a unique opportunity to validate

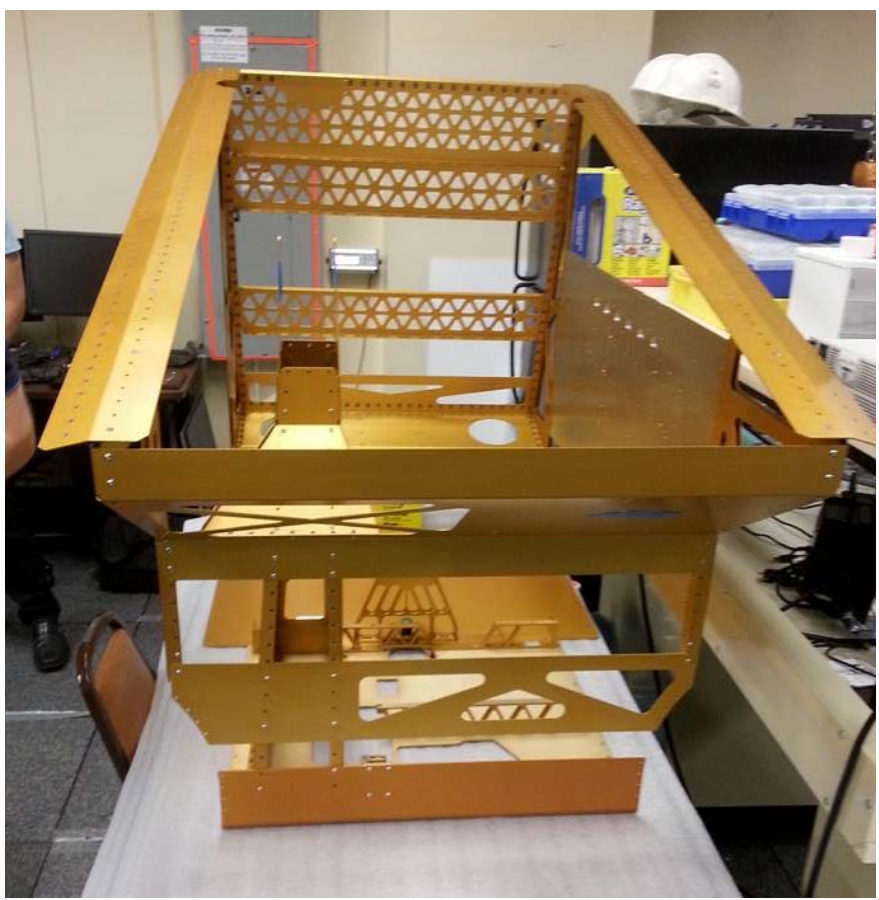

Figure 14. RP15 Rover Frame assembly

core mission operations concepts for RP. These include mission operations with a fully distributed team, waypoint driving with a distributed team, procedures jointly developed with the MOS/flight teams and used for both Integration \& Test, and mission operations, design and maturation of mission tools through actual use, using the builders as the operators, and using low-fidelity paper simulations as a design tool.

RP operations are planned to be fully distributed, with all key mission operators working from their home institutions. This approach provides significant savings over co-location, by eliminating the cost and time spent traveling, especially during the months of Ops training and simulations. It also provides flexibility in training schedules and adaptability to perturbations caused by the flight system during the integration and test period. Fully distributed operations must be validated through simulation. RP15 provides a unique opportunity, early in project development, to operate both the rover and payload using the distributed team at Ames Research Center (ARC) in California (Figure 15), Johnson Space Center (JSC) in Texas, and Kennedy Space Center (KSC) in Florida (Figure 16).

Compared to a Mars mission, the round trip communication latency for a lunar mission is short. It does not however, provide for real-time command and control. We expect the round trip command and control time to vary from six seconds to over 25 seconds for RP. This does allow for smaller granularity decision cycles for driving and near real-time command and control. The RP15 rover will be driven using waypoint driving. The driver and codriver are located at ARC, while "Rover Systems" is located at JSC. The mission command position is at ARC. The command allocations and operational decisions between these positions are being refined bi-weekly through procedural simulations, which increase in fidelity with each sim. We started with procedures and paper, practicing voice calls for each procedure step, and then added data using the rover Processor-In-the-Loop (PIL). Starting with our baseline ops concept of driving commands sent from ARC, systems commands were initiated at JSC and then sent procedurally by an ITOS command station at ARC. We have refined our mission Concept of Operations with each simulation using simulation for design. Rather than holding a series of meetings, we start with a baseline concept, then repeatedly test and refine through simulation. 


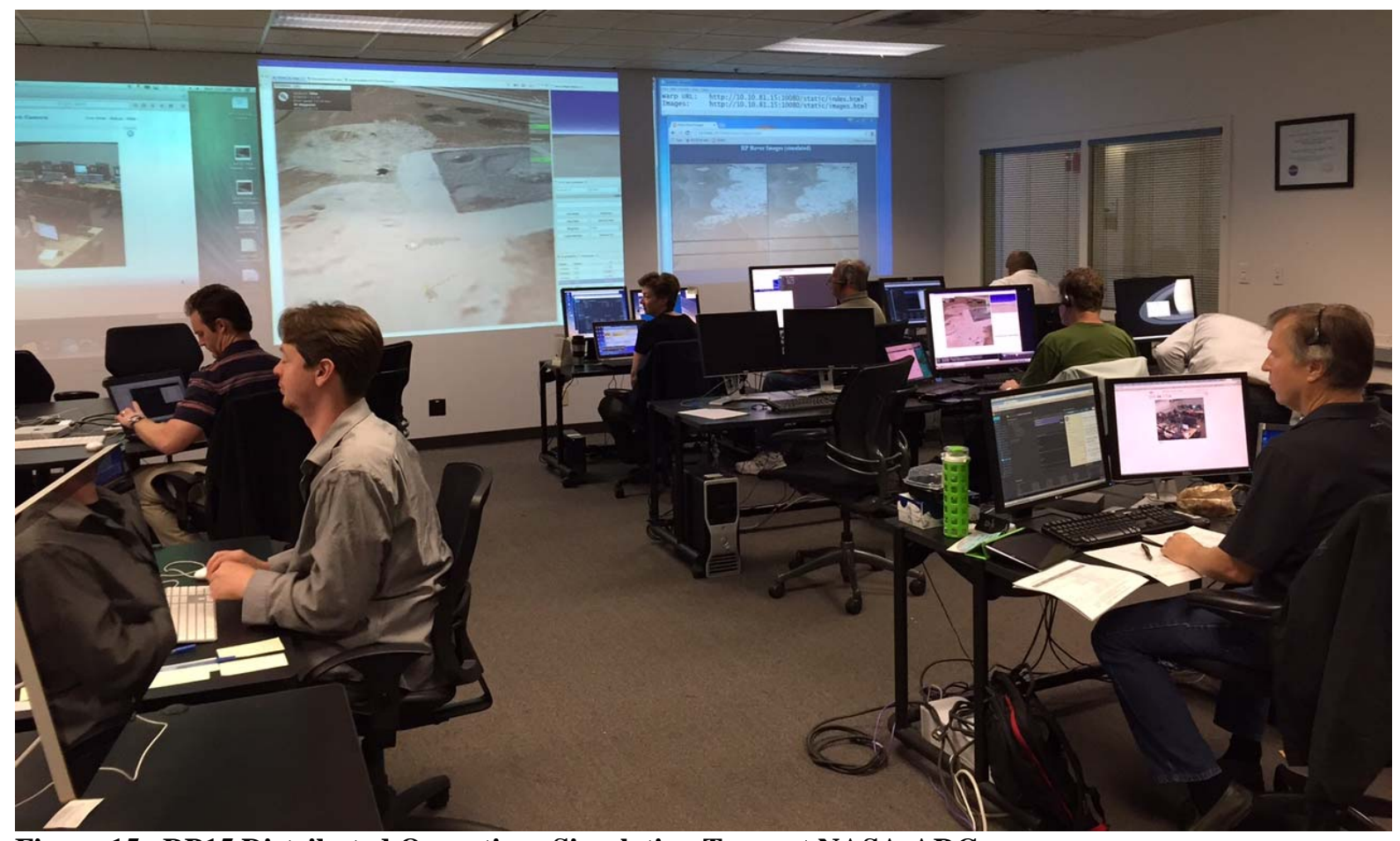

Figure 15. RP15 Distributed Operations Simulation Team at NASA-ARC

Our operational concept provides for a small core mission operations team of a handful of people. Most of the operators for the mission will be drawn from the team building the rover, payload and science instruments. Procedures are designed and tested through use during integration and test, and validated for mission operations in simulation. We are using this process for RP15, eliminating costly walls between development and operations, and maturing and validating procedures through use.

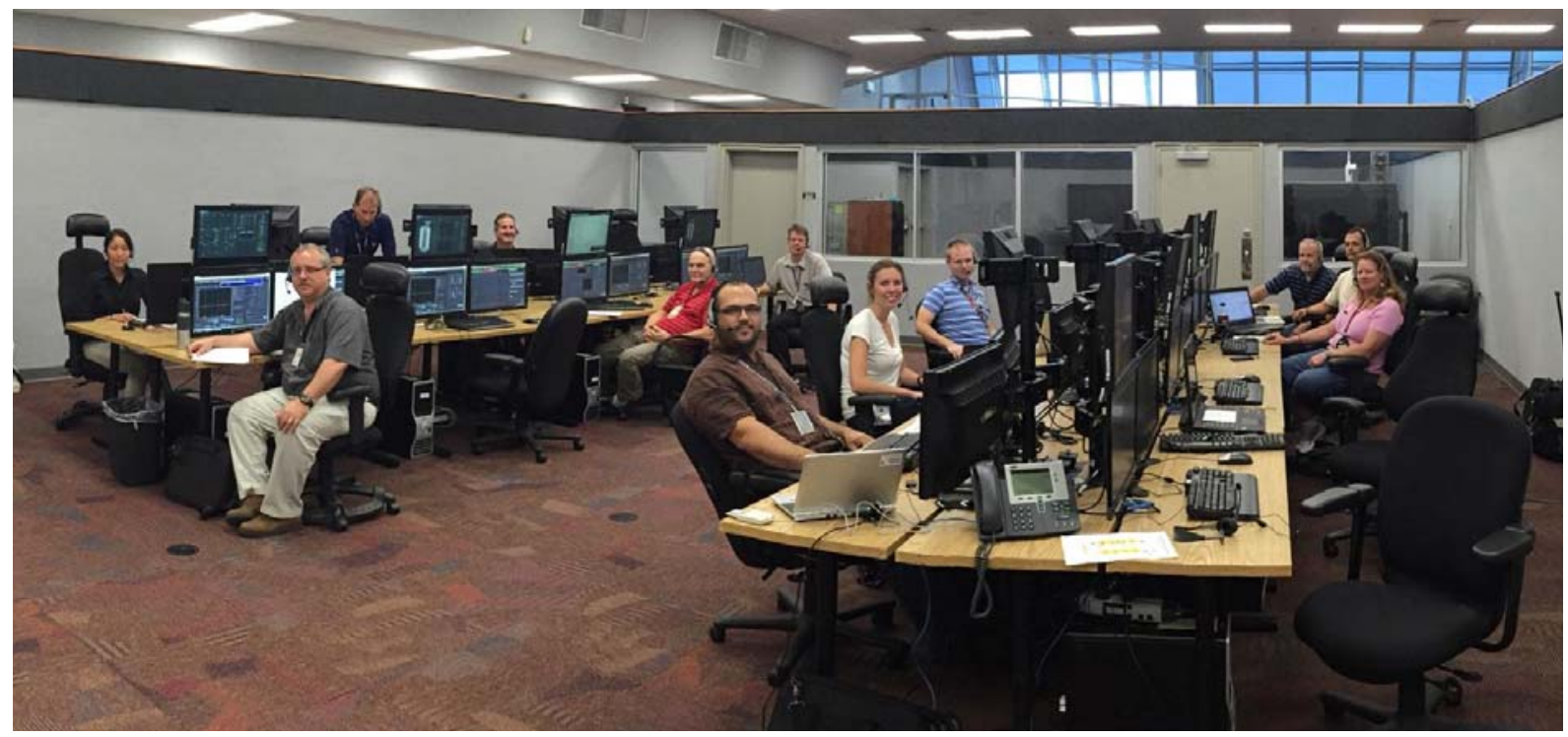

Figure 16. RP15 Distributed Operations Team Simulation at NASA-KSC

Mission operations tools are also following the maturation-through-use philosophy. Rover driving tools and web-based mission operations tools for distributed situational awareness are being designed and tested in integration 
and test, flight software development and procedural simulations. While we think of simulations primarily as a training tool, we are also using sims as a design tool, iteratively refining and trying mission plans, procedures and tools, de-briefing the results, and refining processes and tools ${ }^{[9]}$.

\section{RP15 Integration Status}

Once the principal subsystem Elements were assembled and tested, payload and rover began system-level integration in preparation for the commencement of testing in July-September, 2015. Figures 17-19 show the impressive progress this team has made from concept to assembled hardware by the time of this writing.
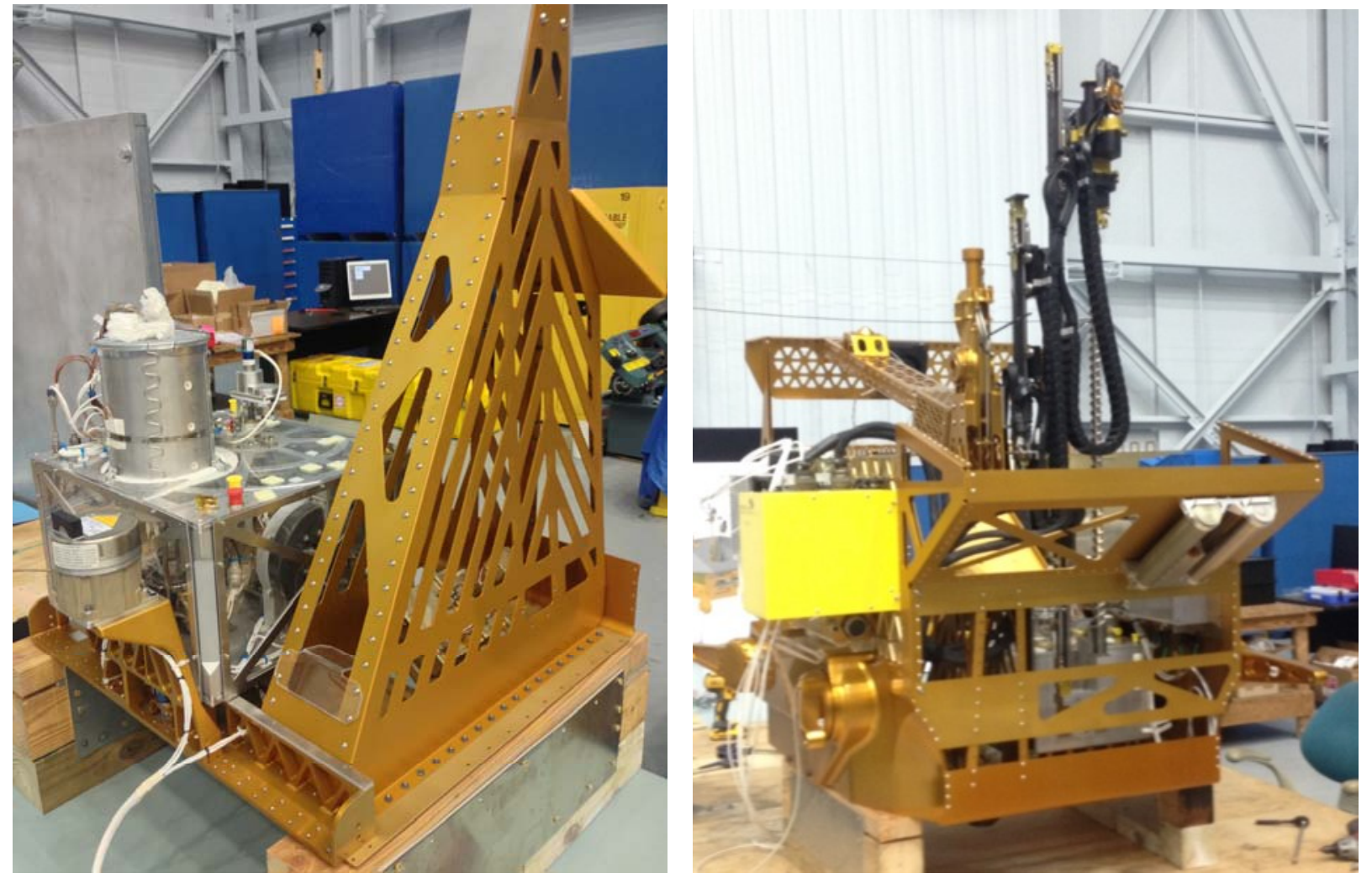

Figure 17. RP15 Payload OVEN system installed on Figure 18. RP15 with Drill installed the rover frame

\section{RP Programmatic Status}

RP is in Phase A Formulation, having successfully passed its NASA Mission Concept Review (MCR) in the Fall of 2013. It is actively involved in international partnership discussions, to maximize return on this novel mission, within budgetary constraints. NASA will be providing the ISRU payload instrumentation, including drill, the roving mobility platform and launch vehicle. The lander will be provided through an international partnership and will likely include additional instrumentation increasing the scientific value of the mission. The mission is scheduled for a 2020 launch, and NASA is working to a cost-effective \$250M budget (not including the launch vehicle).

The NASA stakeholders have classified this mission as "Class D", which is the most risk-tolerant mission classification currently at NASA; this was the same mission classification as LCROSS ${ }^{[8]}$, which successfully rewrote the history books regarding water and the moon. RP looks to extend those cost-efficient, risk-tolerant approaches to a larger mission, while answering fundamental questions to enable future human exploration.

The great work of this RP team can be followed on the web at: www.nasa.gov/resource-prospector and on Twitter @NASAexplores. 


\section{Conclusion}

Resource Prospector is being developed in conjunction with international and commercial partners to deliver an ISRU mobility platform to a polar region on the moon. RP will be the first ISRU demonstration on another planetary body, taking first steps to be able to "live off the land". RP15 is a Phase A "deep dive" ETU development, moving from concept to working hardware roving in a lunar-analogue rock yard in a single year. Through subsequent testing in 2016, RP15 will help reduce risk and improve resource prospector designs and approaches.

\section{Acknowledgments}

The authors would like to thank the hard-working and immensely capable Resource Prospector team, across the many participating NASA Centers. Simultaneously working a Phase A mission towards flight, while also taking a terrestrial ETU rover/payload system from concept, design, build, test, and operations is an impressive feat. This work greatly reduces mission-level risk and matures concepts well beyond Phase A maturity. Additionally, we would like to express gratitude to our customers within the Advanced Exploration Systems Division of NASA-HEOMD as well as the Game Changing Division of NASA-STMD, who asked this team to step-up to this important challenge, while encouraging lightweight approaches to mission management.

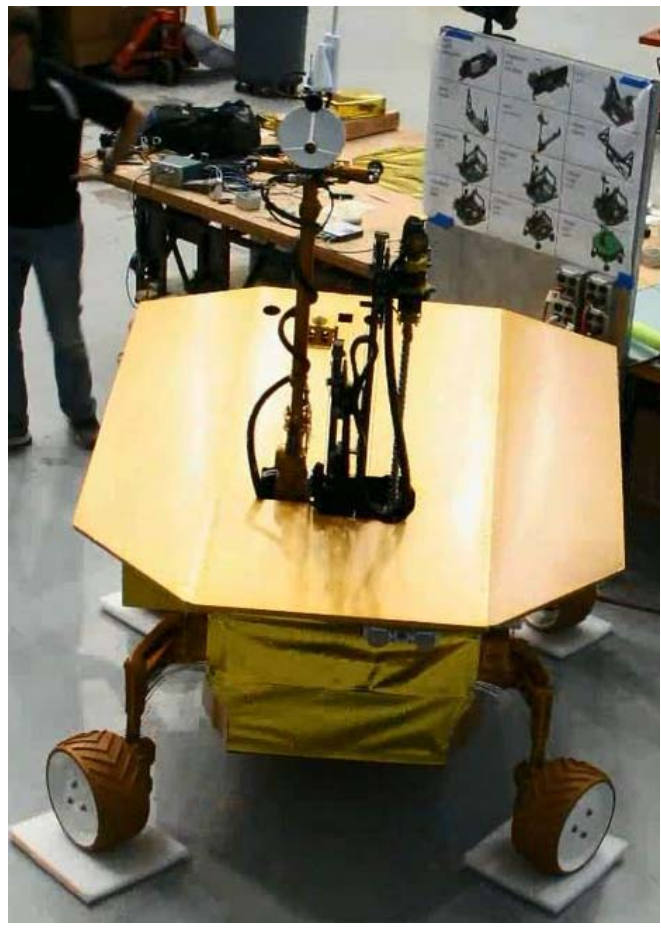

Figure 19. RP15 Integration Complete

\section{References}

1 Andrews, D., Colaprete, A., Quinn, J., Chavers, D., Picard, M., “Introducing the Resource Prospector (RP) Mission”, American Institute of Aeronautics and Astronautics (AIAA) SPACE 2014, San Diego, CA, USA.

2 “The Global Exploration Roadmap,” International Space Exploration Coordination Group (ISECG), September 2013, URL: http://www.globalspaceexploration.org/wordpress/wp-content/uploads/2013/10/GER_2013.pdf, Pg23, [cited 29 May 2014 ].

${ }^{3}$ Zacny, K., Paulsen, G., Chu1, P., Mellerowicz, B., Yaggi, B., Klein-Henz, J., Smith, J., "The Icebreaker Drill System: Sample Acquisition and Delivery for the Lunar Resource Prospecting Mission”, Lunar and Planetary Science Conference (LPSC46), 2015, Woodlands, TX, USA.

${ }^{4}$ Harrison, et al, “Next Generation Rover for Lunar Exploration,” IEEE Aerospace Conference, Big Sky, MT, 2007.

5 Pedereson, L. et al, "Tele-operated lunar rover navigation using LIDAR, ” International Symposium on Artificial Intelligence, Robotics and Automation in Space (I-SAIRAS); Turin; Italy, 4-6 Sep. 2012;

${ }^{6}$ Alstrom, et al, "Robonaut 2 on the International Space Station: Status Update and Preparations for IVA Mobility” American Institute of Aeronautics and Astronautics (AIAA) SPACE 2013, San Diego, CA, USA.

${ }^{7}$ Wilmot, J., "A core plug and play architecture for reusable flight software systems," Space Mission Challenges for Information Technology, 2006. SMC-IT 2006. Pasadena, CA, 2006.

${ }^{8}$ Andrews, D. R., "LCROSS - Lunar Impactor: Pioneering Risk-Tolerant Exploration in a Search For Water on the Moon," $7^{\text {th }}$ International Planetary Probe Workshop (IPPW-7), Barcelona, Paper IAC-11-A5.1.4, 2011.

9 Trimble, J, "Lean Mission Operations Systems Design - Using Agile and Lean Development Principles for Mission Operations Design,” American Institute of Aeronautics and Astronautics, Space Operations Conference 2014, Pasadena, CA 